\title{
Zur Physiologie der vorderen und der hinteren Zentralwindung.
}

\author{
Von
}

\section{Lewandowsky und A. Simons.}

(Mit 2 Textfiguren.)

Die anatomischen Differenzen zwischen der vorderen und der hinteren Zentralwindung stehen fest. Indessen sind weder aus den zellbistologischen noch aus den faseranatomischen Arbeiten bindende Schlüsse auf die Art der beiden Windungen, der vorderen und der hinteren, zu machen.

Die funktionellen Unterschiede können nur durch physiologische und klinische Untersuchungen ermittelt werden. Was die klinischen Ergebnisse, die bisher vorliegen, betrifft, so geht daraus zwar mit völliger Sicherheit hervor, dass die hintere Zentralwindung in einer besonderen Beziehung zur Sensibilität, die vordere zur Motilität steht, aber ob und inwieweit eine völlige Scheidung der motorischen und der sensiblen Funktionen durch die Zentralfurche gegeben ist, ist für die motorischen Funktionen mindestens zweifelhaft und für die sensiblen Funktionen kann mit grosser Wahrscheinlichkeit gelten, dass ihre Lokalisation nach vorn über die Zentralfurche hinübergreift.

Die physiologischen Versuche könnten einmal in den Prüfungen der Ausfallerscheinungen nach Exstirpation der vorderen Windung einerseits, der hinteren andererseits bestehen. In dieser Richtung liegen, worauf wir zurückkommen werden, noch keine bindenden Versuche vor.

Hingegen wird von vielen ein entscheidender Wert gelegt auf die Ergebnisse der elektrischen Reizung, nachdem Grün baum und Sherrington nachgewiesen haben, was vorher Hitzig schon an einem Affen gefunden hatte, dass bei Reizung mit schwachen Strömen nur von der vorderen Zentralwindung Bewegungen ausgelöst werden können. Es sind diese Resultate, die ja auch mit den früheren experimentellen Ergebnissen von Beevor und Horsley 
und anderen nicht übereinstimmen, jedoch nicht überall anerkannt und zuletzt noch von $\mathrm{R}$ oth $\operatorname{man} \mathrm{n}^{1}$ ) entschieden bestritten worden.

Denn es ist Tatsache, dass auch von der hinteren Zentral windung aus Bewegungen, wenn auch mit mehr oder weniger starken Strömen, ausgelöst werden können, und es ist unzweifelhaft richtig, dass es eine petitio principii bedeutet, wenn man allein auf die Tatsache; dass die vordere Zentralwindung leichter erregbar ist, die $\mathrm{Be}-$ hauptung aufstellt, dass nur die vordere erregbar wäre. So hat denn auch vor kurzem $0 . \mathrm{Vog} t$, der ebenfalls die hintere Zentralwindung mit schwächeren Strömen elektrisch unerregbar fand ${ }^{2}$ ), ausdrücklich erklärt, dass er niemals den Gyrus centralis anterior als rein motorisch und den Gyrus centralis posterior als rein sensorisch hingestellt habe ${ }^{3}$ ).

Überlegen wir uns zunächst, a uf welche Weise überhaupt von der hinteren Zentral windung aus Bewegungseffekte bervorgebracht werden können. Als selbstverständlich wird dabei vorausgesetzt, dass die von der vorderen Zentralwindung ausgelösten Bewegungseffekte durch die Projektionsfasern dieser vorderen Windung und insbesondere durch die Pyramiden übertragen werden. Die erste Möglichkeit ist offenbar die, dass Stromschleifen von der hinteren auf die vordere Zentralwindung oder deren Projektionsfasern übergehen; die zweite, dass Assoziationsfasern der hinteren Zentralwindung mit der vorderen verbunden und die Erregung von der binteren auf die vordere und, durch diese Assoziationsfasern vermittelt, dann auf den Projektionsfasern der vorderen zur Peripherie geleitet wird. Eine solche Verbindung müsste wohl auch eine Rolle spielen in der Hypothese Sherrington's, dass Bahnungsvorgänge bei Reizung der vorderen und hinteren Zentral; windung vorkommen. Die zuletzt erwähnte Möglichkeit würde jeden= falls also auf dem Vorhandensein präformierter anatomischer Wege beruhen. Es würde die Auslösung von Bewegungen von der hinteren Zentralwindung aus dann das Analogon eines normalen physiologischen Vorganges sein, während die erste Vorstellung ja einfach aus der Nichtausschaltung einer Fehlerquelle sich ergeben würde. Endlich

1) Über die physiologische Wertung der kortikospinalen (Pyramiden-)Bahn. Arch. f. Anat. u. Physiol. 1907.

2) Journ. f. Psychol. u. Neurol. Bd. 8. 1907. Zur Kenntnis der -elektrisch erregbaren Hirnrindengebiete usw. (dort Literaturzusammenstellung).

3) Verhandl. d. Berliner Gesellsch. f. Psychiatrie u. Nervenkrankheiten. Sitzung vom 11. Nov. 1907. 
würde eine dritte Möglichkeit darin bestehen, dass die hintere Zentralwindung eine eigene Projektionsfaserung besässe, und das wird wohl von den meisten, die für die motorische Funktion der hinteren Zentralwindung eintreten, auch angenommen.

Nun gibt es offenbar eine Methode, die die Möglichkeiten 3 und 1 ausschaltet. Das ist nämlich die Reizung der hinteren Zentralwindung nach Exstirpation der vorderen und Degeneration von deren Projektionsfasern. Die Exstirpation der vorderen Zentralwindung mit sofort nachfolgender Reizung der hinteren hat offenbar deswegen keinen Sinn, weil etwaige Stromschleifen dann noch leitungsfähige Elemente treffen. Nur wenn die Projektionsfasern der vorderen Zentralwindung schon degeneriert sind, so müsste man den Erfolg auch noch so starker Ströme als beweisend fürdieelektrisehe Erregbarkeit der binteren Windung ansehen.

Auch diese Methode würde nicht zum Ziele führen, wenn eine Behauptung von C. und $\mathrm{O} . \mathrm{V}_{\mathrm{g}} \mathrm{gt}^{\mathrm{l}}$ ) richtig wäre, dass nämlich die Reizung der Narbe der vorderen Zentralwindung auch 3 Wochen nach der Operation, also wenn die Degeneration der Pyramidenfasern in vollem Gange ist, noch motorische Effekte hätte. Es müssten ja dann unter allen Umständen bei genügender Verstärkung des Stromes Reize von der hinteren Zentralwindung aus auch auf die erregbaren Projektionsfasern übergehen können. Wir müssen jedoch auf Grund unserer Versuche die erwähnte Behauptung Vogt's, die ja auch allen bekannten Tatsachen über das Leitungsvermögen degenerierter Fasern widerspricht, entschieden bestreiten. Ihre Ergebnisse sind nur dadurch zu erklären, dass sich das erregbare Gebiet nicht völlig exstirpiert haben und also bei Reizung der Narbe Stromschleifen auf stehengebliebene Reste der erregbaren Rinde oder deren Projektionsfaserung übergingen. Wir fanden schon nach 14 Tagen die Narbe völlig unerregbar (und wahrscheinlich tritt diese Unerregbarkeit ja auch schon früher ein).

Ehe wir über unsere Versuche des weiteren berichten, muss noch erwähnt werden, dass einige Versuche bereits von Sherrington und Grünbaum ${ }^{2}$, ferner von Vogt's und Rothmann ${ }^{8}$ ) vor-

1) l. c.

2) Observations on the physiology of the cerebral cortex of some of the higher apes. Proceed. of the Roy. Soc. vol. 69. 1901.

3) 1. c. 
liegen. Und zwar hat Rothmann nach Exstirpation der vorderen Zentralwindung positive Resultate von der hinteren erzielt. Von Sherrington und Grünbaum und Vogt's wird die prinzipielle Bedeutung dieser Versuchsanordnung nicht genügend gewürdigt. Keiner von ihnen hat mit stärksten Strömengereizt. Die Stromstärke Vogt's bewegt sich in den Versuchen über diese Frage zwischen 90-80, einmal bis 70 R.-A.; über die Stromstärken, die Sherrington angewandt hat, haben wir keine genaueu Angaben gefunden. Er drückt sich nur so aus, dass Zerstörungen der vorderen Zentralwindung die Erregbarkeit der hinteren nicht vermehre. ("Destruktion of the pre-central convolution does not make in the more possible to obtain movements under faradisation from the post-central convolution. ${ }^{2}$ )

Über die Technik unserer Versuche an zehn Affen (Macacus Rhesus und einer Meerkatze) ist wenig zu sagen. Die Operationen wurden so ausgeführt, dass wir nach Freilegung der Zentralfurche mit zwei Spateln die vordere und die hintere Zentralwindung möglichst bis zum Grunde der Furche voneinander abdrängten und dann von hinten nach vorn die vordere Zentralwindung meist bis weit hinein in den Stirnlappen entfernten. Dabei kamen wir uatürlich in die Projektionsfaserung der vorderen Zentralwindung. Die Untersuchung mikroskopischer Schnittpräparate hat gezeigt, dass bei diesem Vorgehen gröbere Verletzungen der hinteren Zentralwindung und ihrer Projektionsfasern vermieden werden können. Auch haben uns Kontrollversuche bewiesen, dass, wenn man in analoger Weise die hin ter e Zentral windung exstirpiert, die Erregbarkeit der vorderen in keiner Weise gestört wird, ebenso wie kleine Reste der vorderen Zentralwindung, die besonders im lateralen Teil der vorderen Zentralwindung in unseren Versuchen manchmal stehen blieben, dicht neben der Narbe in geradezu überraschender Weise ihre Erregbarkeit bewahrt hatten.

Die Reizung selbst wurde 3-6 Wocben nach Exstirpation der vorderen Zentralwindung ausgefuhrt, und zwar bipolar. Die unipolare Reizung bat ja keinen prinzipiellen Vorzug ausser der exakteren Lokalisation; diese kam aber für uns nicht in Frage, und wir hatten bei bipolarer Reizung die Möglichkeit, sehr viel stärkere Ströme anzuwenden, ohne durch die bei solchen Strömen recht unangenehmen Wirkungen des den Körper durchfliessenden Stromes gestört zu werden. Soweit wir sie in einigen Versuchen benutzt 
baben, hat sie uns keine anderen Ergebnisse als die bipolare Reizung geliefert.

Wîr haben Stromstärken bis zu $20 \mathrm{~mm}$ R.-A. des mit einem Akkumulator armierten $d u$ B o is-Rey mond'schen Schlitteninduktoriums verwandt, manchmal noch stärkere Ströme, bis allgemeine Abwehrbewegungen (wohl durch Stromschleifen auf die Dura) resp. Wirkungen auf die andere Hemisphäre eintraten. Wir haben immer versucht, mit ganz geringen Graden der Narkose auszukommen, manchmal auch das Tier ganz aus der Narkose erwachen lassen. Wir haben in dieser Weise an fünf Affen, denen die vordere Zentralwindung ein- bzw. doppelseitig exstirpiert war, die bintere gereizt.

Wir sind dabei zu dem Ergebnis gekommen, dass die hintere Zentralwindung nach Exstirpation der vorderen auch mit den stärksten Strömen völlig unerregbar ist, und die minimalen Wirkungen, die wir bei zwei Versuchen bekommen haben, sind nur geeignet, das eben erwähnte Frgebnis $\mathrm{zu}$ bestätigen, da sie sich durch zurưckgebliebene Reste der vorderen Zentralwindung erklären, wie wir bei der Sektion des Tieres feststellen konnten. Denn es ist selbstverständlich unmöglich, die Operation so exakt auszuführen, dass im ganzen Verlauf der Zentralfurche die vordere Zentralwindung immer gerade bis auf den Grund exstirpiert wird, und zwar mit völliger Schonung der hinteren.

In den Präparaten dieser Fälle, die wir mikroskopisch untersuchten, sind vielmehr in der Tiefe der Furche noch Reste der vorderen Zentralwindung erhalten, übrigens auch in einem Falle, in dem wir eine elektrische Erregbarkeit nicht mehr fanden. Man muss in diesem Falle annehmen, dass entweder diese kleinen Reste nicht mehr fähig waren, die elektrisehe Erregung zu vermitteln, oder aber, und das ist wahrscheinlicher, dass die Projektionsfasern dieser Reste doch völlig durchschnitten waren.

Gerade die Ergebnisse in solchen Fällen, wo selbst kleine makroskopische Reste der vorderen Zentralwindung zurückgeblieben waren und schon dadurch die Schonung der hinteren Windung gesichert war, zeigen am deutlichsten, dass von einer selbständigen Erregbarkeit der hinteren Zentralwindung nicht die Rede sein kann.

Wir bilden das Gehirm eines Affen ab (Fig. 1), bei dem der lateralste Teil der vorderen Zentralwindung noch erhalten, aber 
schwer verletzt war, bei dem dann medialwärts zwar die gauze Kuppe der Zentralwindung exstirpiert ist, aber sich doch in der Tiefe noch der Grund der Zentralfurehe wahrnehmen lässt. Hier liessen sich vom unteren Teil der stehengebliebenen vorderen Zentralwindung mit Leichtigkeit Facialis- und Kaubewegungen auslösen (bei 1, 2 der Fig. 1); die hintere Zentralwindung war im weiteren Verlaufe fast völlig unerregbar. Nur vom medialsten Teile, d. h. von der Mantelkante, also vom Parazentrallappen, wo ja die Grenze

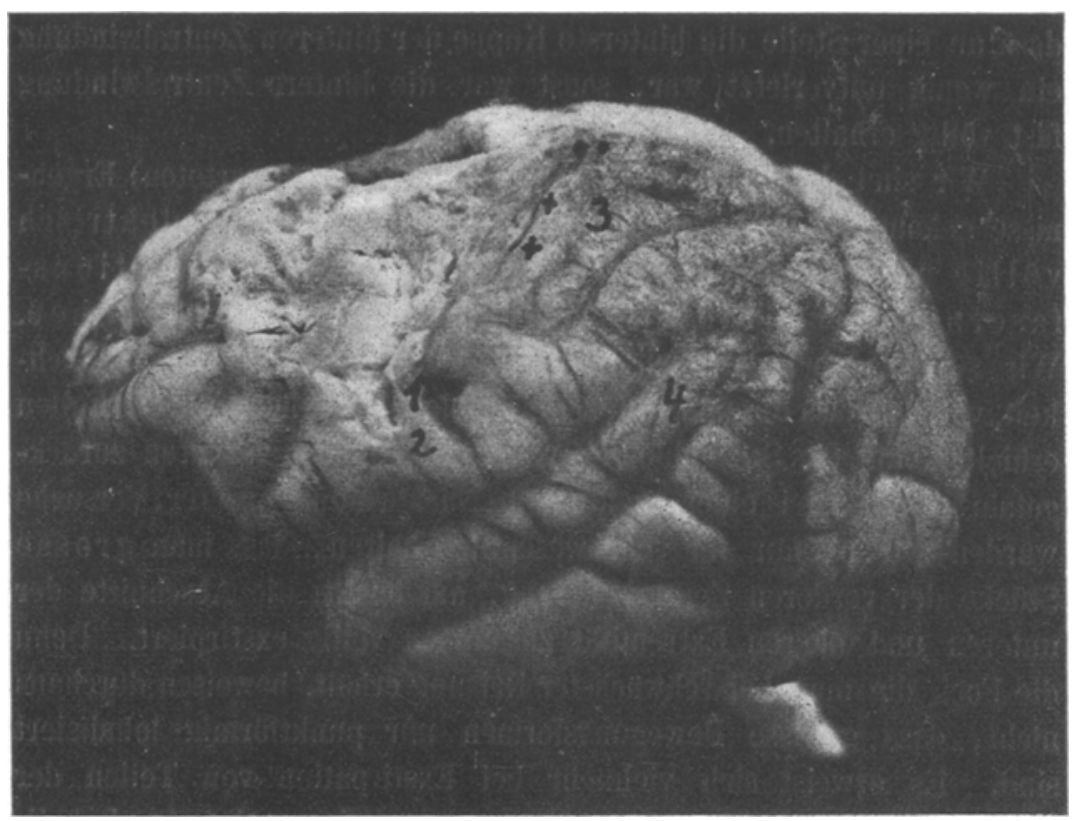

Fig. 1. Über die beiden Punkte an der Mittellinie und die beiden Krenze hinter der Zentralfurche vgl. Text. 1 Reizfeld für den unteren Facialis. 2 Kaubewegungen. 3 Ohrbewegung nach vorn (vgl. Text). 4 Anlegen des Ohres.

zwischen vorderer und hinterer Zentralwindung nicht mehr gezogen werden kann, konnten wir schwache Bewegungen des Beines hervorrufen (die beiden Punkte der Fig. 1) und bei allerstärksten Strömen und nur bei einer so grossen Entfernung der Elektroden voneinander, wie sie verhältnismässig durch die beiden Kreuze der Abbildung angegeben ist, konnten wir ein leichtes Zurückziehen des Armes erzielen. Es ist wohl ohne weiteres anzunehmen, dass diese geringe Erregbarkeit aut die minimalen Reste der vorderen Zentralwindung zurückgeführt werden muss; insofern sind diese Fälle, die trotz 
völliger Erhaltung der hinteren Zentralwindung und sogar von Resten der vorderen Zentralwindung bei Reizung der hinteren nur diese ganz geringen Spuren der Erregbarkeit, niemals etwa isolierte Bewegungen der Hand, des Fingers oder des Daumens zeigten, beinahe beweisender für die Unerregbarkeit der hinteren Zentralwindung als die mit völlig negativen Ergebnissen.

Einen solchen Fall, bei dem wir eine Erregbarkeit der Extremitäten überhaupt nicht mehr nachweisen konnten, bilden wir in Fig. 2 (S. 252) ab. Die mikroskopische Untersuchung hat hier ergeben, dass an einer Stelle die hinterste Kuppe der hinteren Zentralwindung ein wenig mitverletzt war, sonst war die hintere Zentralwindung fast völlig erhalten.

Wir sind demnach zu dem (uns durchaus unerwarteten) Ergebnisse gekommen, dass die hintere Zentralwindung elektrisch völlig unerregbar ist, d. h. keine eigenen Projektionsfasern besitzt, die elektrisch erregt werden könne. Wir setzen dabei die eben begründete sehr grosse Wahrscheinlichkeit voraus, dass die minimalen Reizerfolge, die wir in einigen Fällen erhielten, auf das Stehenbleiben von Resten in der vorderen zurückgeführt sind. Bei einer eventuellen Nachprüfung unserer Versuche würden wir es nur für sehr wesentlich halten, dass man grosse Stücke der vorderen Zentralwindung, am besten die Abschnitte der unteren und oberen Extremität zusammen völlig exstirpiert. Denn die Foci, die man bei schwächster Reizung erhält, beweisen durchaus nicht, dass gewisse Bewegungsformen nur punktförmig lokalisiert sind. Es erweist sich vielmehr bei Exstirpation von Teilen der vorderen Zentralwindung doch eine etwas diffusere Art der Lokalisation, und man kann die stehengebliebenen erregbaren Zentren der vorderen Zentralwindung dann natürlich auch dureh Stromschleifen von der hinteren aus erregen.

Es scheint, dass die innere Konstitution der motorisch erregbaren vorderen Zentralwindung doch wohl eine viel kompliziertere ist, als aus der so einfachen Aufstellung einer Menge von Foci hervorgehen würde. Diese Foci können immer nur als die Stellen der leichtesten Erregbarkeit aber nicht als die einzigen Zentren der Erregbarkeit der einzelnen Bewegungen angesehen werden, und daher genügt auch für die Bestimmung der Bedeutung der vorderen und der hintereu Zentralwindung nicht das Herausschneiden kleiner Stücke. 
Fs ist nun fraglich; was das Fehlen der elektrischen Erregbarkeit der hinteren Zentralwindung für ihre physiologische Funktion bedeutet. Dass die hintere Zentralwindung auf dem Wege von Assoziationsbahnen auf die vordere einwirken kann, ist wohl sicher, jedenfalls wird, wie bemerkt, diese Frage durch unsere Versuche nicht berührt. Aber auch das lässt sich denken, dass eine Hirnregion, die für èlektrische Ströme nicht mehr erregbar ist, doch noch eine willkürliche Bewegung auf dem Wege eigener Projektionsfasern vermitteln könnte. Diese Fragen würden nur Exstirpationen der vorderen und der hinteren Zentralwindung entscheiden. Solche Versuche sind bisher systematisch nicht angestellt worden. Insbesondere geht $\mathrm{H}$. $\mathrm{Munk}$, der ja wohl solche Versuche angestellt haben muss, über diese Frage hinweg, wenn sich auch aus seinen Abhandlungen ergibt, dass er einen Unterschied zwischen vorderer und hinterer Zentralwindung nicht anerkennt. Brodmann ${ }^{1}$ ) hat acht Affen operiert (je vier mit exstirpiertem. Gyrus centralis anterior resp. Gyrus centralis posterior). Die beiden von ihm als Beispiele hierfür vorgestellten Tiere befanden sich $5-6$ Tage nach der Operation.

„Beide Tiere gebrauchten übereinstimmend ihre rechtseitigen Gliedmaassen zu intendierten Einzelbewegungen überhaupt nicht".... "Ein deutlicher Untersehied aber war in der Ruhehaltung und bei Lokomotion zu beobachten", derart, dass das Tier nach Operation der vorderen Zentralwindung auch hierbei schwerer geschädigt erschien, während der andere Affe dabei sich fast normal verhielt. Nun ist aber bekannt und Brodmann hat das in der Diskussion zu seiner Demonstration (1. cit.) zugegeben, dass gerade diese Störung der Lokomotion sich nach nicht zu langer Zeit auch bei ausgedehnten Zerstörungen zurückbilden kann, so dass aus den Brodmann'schen Versuchen nur ein quantitativer Unterschied in bezug auf die Fortbewegung und gar keiner in bezug auf die Auslösung der Einzelbewegungen hervorgehen würde. Es erseheint uns sicher, dass man, da unserer Ansicht nach (die freilich nicht von allen geteilt wird) die eine Hemisphäre für die andere bis zu einem gewissen Grade eintreten kann, in dieser Frage nur mit doppelseitigen Exstirpationen zu definitiven Ergebnissen kommen wird. Wir haben einige orientierende Versuche in dieser Richtung angestellt.

1) Neurol. Zentralbl. Bd. 24 S. 1158. 1905. 
Wir häben drei Affen doppelseitig operiert. Dem einen waren beide vorderen Windungen bis weit in den: Stimlappen, dem anderen beide hinteren Windungen zugleich mit einem Teil des Gyrus angularis entfernt, so dass also hier das ganze Gebiet zwischen Zentral- und zwischen Affenfurche fehlte. Eudlich haben wir dem dritten Tiere auf der einen. Seite die hintere, auf der anderen Seite die vordere Zentral windung exstirpiert.

Zunächst haben wir die Beobachtung gemacht, dass nach Exstirpation einer hinterenZentralwindung (bei intakter anderer Hemisphäre) der Affe durchaus nicht sofort die Fähigkeit einbüsst, isoliertè Bewegungen mit dem kontralateralen Arm zu machen. Besonders gleich nach der Operation versuchte der Affe mit dem kontralateralen Arm wie bisher zuzugreifen. Die Beobachtung von Brodmann, dass die Einzelbewegungen sowohl nach Extirpation der vorderen als auch der hinteren verloren gingen, bedarf also der Ergänzung; dass ein Tier nach Exstirpation der vorderen Zentralwindung noch Einzelbewegungen macht und erst später auf sie verzichtet, kommt eben nicht vor. Was nun die Bewegungen nach Exstirpation der hinteren Zentralwindung betrifft, so sind sie ausserordentlich ataktisch, sie erreichen ihr Ziel nicht, und sehr bald verzichtet dann der Affe darauf, diese Hand zu isolierten Bewegungen zu benutzen, ausser wenn es gilt sehr grosse Stücke festzuhalten, für die eine Hand nicht genügt; gelegentlich sieht man aber immer auch noch hier eine isolierte Bewegung. Sicherlich ist aber von vornherein eine erhebliche Sensibilitätsstörung vorhanden. In die Hand gelegte Gegenstände werden bei Ausschluss des Gesichtssinnes nicht ergriffen, geringe Berührungen werden nicht beachtet, auch Kälte (Wattebausch mit Äther) anscheinend nicht unangenehm empfunden, dagegen lösen schmerzhafte Reize, wenn auch wohl schwerer wie auf der anderen Seite, eine Allgemeinreaktion aus.

Die Bewegungsstörung, die auf diesem Arm besteht, tritt nun sofort und am besten hervor, wenn man auf der anderen Seitedievordere Hemisphäre exstirpiert, der Affe zögerte dann nicht, sofort den bisher nicht benutzten Arm zu gebrauchen. Die Bewegungsstörung bei diesem Affen war denn auch einseitig die gleiche wie bei einem Affen, dem wir die beiden hinteren Zentralwindungen exstirpiert hatten und bestand in einer hochgradigen Ataxie.

Der Affe ohne hintere Zentral wind ungen kletterte schon am Tage nach der Operation; trotzdem vermied er es häufig, die 
Hände zum Greifen von Nahrung zu benutzen, er beugte sich vielmehr zu diesem Zwecke soweit vor, dass er die Nahrung mit dem Maule vom Boden fassen konnte; immerhin griff er häufig genug zu, und dann sah man, dass jede Feinheit der Bewegung verloren gegangen war. Fr fasste den Reis nicht mit den Fingern, sondern mit der Vola und sehob ihn so sehr ungeschickt ins Maul. Manchmal liess er auch Mohrrübenstücke wieder fallen; ebenso zeigte er sich sehr ungeschickt beim Durchgreifen durch die Gitterstäbe.

Typisch war sein Verhalten, wenn man ihm eine Rübe an seinen Käfig so hinlegte, dass er sie nicht sehen konnte. 'Er griff dann durch die Gitterstäbe hindurch nach der Stelle, wo, wie er gesehen batte, die Rübe hingelegt war. Er erreichte sie wohl auch trotz der Ataxie, schloss dann aber nicht rechtzeitig die Faust, fuhr mit dem Arme hin und her, und es gelang ihm dann auch nicht ein einziges Mal, die Rübe zu erfassen. Da er dazu durchaus imstande war, wenn er einen Gegenstand sah, so kann der Grund des Versagens hier wohl nur in einer Sensibilitätsstörung belegen sein, darin also, dass er den Gegenstand mit dem Tastsinn nicht genügend erkennen konnte.

Eine motorische Störung in dem Sinne einer Parese war bei dem Tiere nie vorhanden, und man hatte auch von vornherein den Eindruck, dass ihm auch die Einzelbewegung als solche nicht etwa schwer wurde; auch sie war vielmehr nur im hohen Grade ataktisch. Auch die Gemeinschaftsbewegungen waren etwas ataktisch, aber lange nicht so wie die Einzelbewegungen. In der Ruhe sass er vornüber geneigt, zeigte aber keine ersichtlichen Gleichgewichtsstörungen, auch nicht sofort nach der Exstirpation. Sehen und hören war bei diesem Affen ungestört, und er überlebte die Exstirpation in der zweiten Hemisphäre sechs Wochen.

Ein völlig anderes Bild bot nun der Affe, dem wir die beiden vorderen Zentral windungen exstirpiert hatten. Nach der zweiten Operation lag der Affe auf der Seite, vermochte nicht sich aufzurichten; zeigte man ihm eine Banane, so öffnete er das Maul und bewegte den Kopf etwas der Banane entgegen; wurde er beunruhigt, so versuchte er sich aufzurichten, was ihm aber nicht gelang. Nur den Kopf vermochte er ein wenig zu erheben. Die Extremitäten machten nur strampelnde Bewegungen; nach dem Gitter des Käfigs griff er und hielt es fest. Zwei Tage nach der Operation konnte er so an der Seitenwand des Käfigs sich etwas 
aufrichten. Wenn man ihn an der Rückenhaut festhielt, so dass die Extremitäten herunterhingen und den Boden berührten, machte er Gehbewegungen, fiel aber sofort auf die. Seite, wenn man ihn losliess. Von isolierten Bewegungen mit Ausuabme des Sehliessens der Hand um einen in die Hand gelegten Gegenstand war keine Rede. Der Affe lebte 8 Tage nach der zweiten Operation und starb, weil er spontan nicht Nahrung nahm und künstlich nicht genügend ernährt werden konnte. Nahrung, die man ihm auf die Zunge legte, wurde noch am nächsten Tage im Maul gefunden. Selten schluckte er einmal, und von einem Kauen war keine Rede.

Es ist uns übrigens nicht bekannt, dass Fressstörungen von solcher Schwere bisher überhaupt experimentell beobachtet sind. D. Franck ${ }^{1}$ ) hat an einem Affen besonders die Gegend des Fusses der Zentralwindung und des Opereulum (hintere Grenze Fossa Sylvii, vordere Grenze etwas über die Präzentralfurche hinaus). exstirpiert und schwere Störungen der Nahrungsaufnahme, aber keine Schlingstörungen beobachtet. Es scheint also, dass auch zur Erzeugung schwerster Störungen der Fressfunktionen die Herausnahme sehr grosser Stücke notwendig ist.

Im Gegensatz also zu dem Affen mit beiden exstirpierten hinteren Zentralwindungen bot dieses Tier das Bild einer hochgradigen motorischen Störung. Wenngleich wir nur über diese beiden Versuche doppelseitiger Exstirpationen verfügen, so glauben wir doch aus ihnen im Zusammenhang mit den Beobachtungen an einer Reihe einseitig operierter Affen schliessen zu können, dass nicht etwa nur quantitative, sondern qualitative wesentliche Differenzen der Funktion beider Zentralwindungen bestehen.

Es scheinen also die Exstirpationsversuche, au deren Fortsetzung wir verhindert sind, jedenfalls nicht gegen die durchaus verschiedene Wertigkeit der vorderen und der hinteren Zentralwindung und insbesondere für die vorwiegende und alleinige Bedeutung der vorderen Zentralwindung für die Motilität $\mathrm{zu}$ sprechen und somit unsere Reizungsergebnisse zu bestätigen.

Wir haben nun bei unseren Versuchen noch einige Nebenbeobachtungen gemacht, die wir hier kurz zusammenstellen wollen.

1) Arch. f. Physiol. 1900 S. 209. 
1. Wir sahen in zwei Fällen, also nicht regelmässig, von der hinteren Zentralwindung, und zwar von einem ausgedehnteren Feld, das in Fig. 1 mit 3 bezeichnet ist, trotz - in einem Falle völliger, im anderen fast vollstäıdiger - Aufhebung aller anderen Bewegungen schon bei schwächeren Strömen eine ganz besondere Form der Ohrbewegung, derart, dass das Ohr der Gegenseite sich gewissermaassen nach vorn zusammenrollte.

Die Ohrbewegungen bei Reizung des Temporallappens (Fig. 2), die wir bestătigen konnten, haben eine ganz andere Form als die von uns beobachtete und eben beschriebene Bewegung.

2. Vom Stirnlappen, der in unseren Versuchen mehr oder weniger: stehen blieb, konnten wir Rumpfbewegungen auch bei stärksten Strömen niemals erzielen; auch dann nicht, wenn der vorderste Teil des Gebietes für Rumpf- und Nackenbewegungen stehen geblieben und erregbar, also eine Unterbrechung der Projektionsfaserung vom Stirnlappen wobl ausgeschlossen war.

3. Bei einem Affen haben wir vom Stirnlappen an einer umschriebenen Stelle vor dem Gebiete für die Augen- und Nackenbewegungen bei $30-40 \mathrm{~mm}$ R.-A. so regelmässig $\mathrm{S}$ tim mgebung gehört, dass eine Täuschung in diesem Falle ganz ausgeschlossen war, wenn wir auch den Befund in anderen Fällen niemals wieder erzielen konnten. Die Stimme entsprach ganz dem Krächzen oder Grunzen, das ein Affe so oft hören lässt, wenn er aufgeregt ist.

4. Wir konnten ferner die früher schon von Schäfer u. a. mehrfach gemachte Beobachtung bestätigen, dass auch nach völliger Vernichtung der Augenbewegungszentren im Stirnlappen vom Occipitallappen aus noch konjugierte Seitwärtsbewegungen nach der Gegenseite zu erhalten sind. Diese Bewegungen können also nicht über den Stirnlappen gehen, sondern nur auf eigenen Projektionsfasern des Occipitallappens. Wir bemerken ausdrücklich, dass wir nicht nur Seitwärtswendungen der Augen, sondern auch des Kopfes unter diesen Umständen vom Occipitallappen aus gesehen haben.

Auch die Ohrbewegungen vom Temporallappen lassen sich noch erzielen, wenn die Facialiszentren der vorderen Zentral windung völlig exstirpiert sind, müssen also auch ihre eigenen Projektionsbahnen besitzen.

5. Wir haben nun in einigen Fällen nur die Zone der vorderen Zentralwindung exstirpiert, die nach O. und 
C. Vogt elektrisch erregbar ist, und haben gesehen, dass bei Anwendung stärkerer Ströme doch auch ein nach vorn angrenzender Streifen grauer Substanz noch elektrisch erregbar ist. Auch das Augenfeld von Vog t's reicht im ganzen wohl etwas weiter nach vorn und median, als es nach den Abbildungen Vogt's erscheinen würde: (Fig. 2). Es würde sich wohl verlohnen, die Erregbarkeit innerhalb der motorischen Gebiete nach Exstirpation mehr oder weniger grosser Rindenpartien einmal eingehender zu unterseuhen.

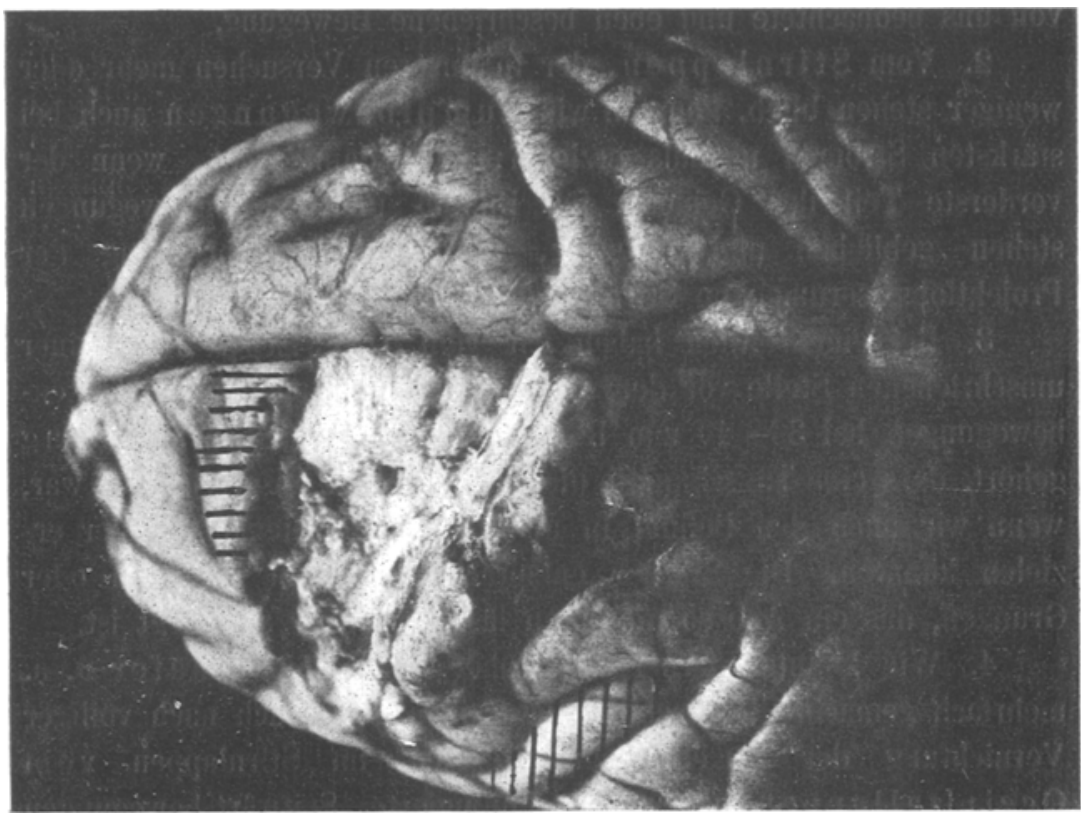

Fig. 2. Von dem wagerecht schraffierten Gebiet wurden konjugierte Augen- und Kopfbewegungen, von dem senkrecht schraffierten Ohrbewegungen ausgelöst.

Was das "A ugenfeld“ Vogt's noch betrifft, so halten wir diese Bezeichnung für nicht korrekt, weil sich regelmässig und meist auch schon mit schwachen Strömen mit den Augenbewegungen zusammen von hier auch Kopfbewegungen auslösen lassen ${ }^{1}$ ), auch nach Exstirpation der hinter dem Augenfeld gelegenen eigentlichen vorderen Zentralwindung.

1) Einmal konnten wir auch (wie Vogt's zweimal am intakten Affen) eine isolierte Seitwärtswendung des Kopfes ohne Augenbewegungen nach Exstirpation eines Teils dieses Feldes erzielen. 
Es liegen also in diesem Augenfeld auch Rindenzentren für die Seitwärtsbewegungen des Kopfes mit eigenen Projektionsfasern; wenn es scheint, als ob Vogt's diese Kopf bewegungen als sekundäre ansähen, so können wir mit diesem von Beevor und Horsley eingeführten Wort einen Begriff nur dann verbinden, wenn damit gesagt werden sollte, dass diese sékundären Bewegungen durch Ausbreitung des Stromes auf benachbarte Gebiete zustande kämen. Das ist in unserem Falle aber völlig ausgeschlossen. Dass man die Kopfbewegungen meist nicht isoliert erzielen kann, beweist pur, dass entsprechend dem engen funktionellen auch ein sehr enger anatomischer Zusammenhang der Zentren für Augen und Kopf besteht. Diese Annahme schliesst auch nicht das Bestehen einer elektrisch erregbaren Zone für die Hals- bzw. Nackenmuskulatur in der vorderen Zentralwindung selbst aus, vielmehr konnten wir eine solche Zone entspreehend den Angaben von Sherringt on auch feststellen, konnten aber von ihr nicht die typische Seitwärtswendung des Kopfes, sondern in erster Linie eine Drehung des Kopfes um die Vertikalachse des Körpers erzielen.

6. Endlich haben wir noch eine Beobachtung $\mathrm{zu}$ erwähnen, die einerseits zu denken gibt über die innere Konstitution der motorisch erregbaren Zone, und die andererseits für die Frage nach der Erregbarkeit der hinteren Zentralwindung einige Aufklärung gibt.

Es ist nämlich bekannt, und besonders auch von Rothmann ${ }^{1}$ ) betont, dass bei unverletzten Affen von der hinteren Zentralwindung besonders leicht Bewegungen des Daumens auslösbar sind. Es ist dies zuletzt noch von R othmann für die selbständige Erregbarkeit der hinteren Zentralwindung verwendet worden. Wir haben nun zweimal die Beobachtung gemacht, dass manchmal isolierte Daumenbewegungen erzielt werden können, ohne jede lokalisierte Reizung, wenn man nämlich einen feuchten Wattebausch auf ein grösseres Gebiet der vorderen Zentralwindung legt und die Elektroden mit diesem Bausch berührt. Das kann aber nur darauf beruhen, dass die Erregbarkeit der hierfür verantwortlichen Zentren eine grössere als die anderer Zentren ist. Es war in diesen Fällen denn auch viel schwerer durch lokalisierte Reizung der vorderen Zentralwindung eine Bewegung des Daumens auszulösen, weil man die Ströme dann, um die Mitreizung anderer Muskeln zu vermeiden, viel genauer

1) 1. c. 
254 M. Lewandowsky und A. Simons: Zur Physiologie etc.

dosieren und lokalisieren musste. Es ist klar, dass bei einer Reizung der hinteren Zentralwindung derselbe Mechanismus in Frage kommen kann, wie bei dem Versuch mit dem Wattebausch, und wir bemerken noch einmal, dass wir nach Exstirpation der vorderen Zentralwindung isolierte Daumenbewegungen oder dergleichen niemals gesehen haben. Die Tatsache der Bestimmung der Reizerfolges durch die verschieden grosse Erregbarkeit einzelner Zentren lässt es auch fraglich erscheinen, ob der Bestimmung von punktförmigen Focis eine solche Bedeutung beizumessen ist, dass sich die hierauf von $\mathrm{O}$. und $\mathrm{C}$. Vogt aufgewendete Mühe anders denn aus reinen morphologischen Gesichtspunkten lohnte, Die physiologische Erregung dürfte doch eben anders vor sich gehen, als wir das durch elektrische Reizung nachahmen können. 